\title{
Upward Wave Activity Flux as a Precursor to Extreme Stratospheric Events and Subsequent Anomalous Surface Weather Regimes
}

\author{
LORENZO M. POLVANI \\ Department of Applied Physics and Applied Mathematics, and Department of Earth and Environmental Sciences, Columbia University, \\ New York, New York \\ DARRYN W. WAUGH \\ Department of Earth and Planetary Sciences, The Johns Hopkins University, Baltimore, Maryland
}

(Manuscript received 2 June 2003, in final form 12 January 2004)

ABSTRACT

\begin{abstract}
It has recently been shown that extreme stratospheric events (ESEs) are followed by surface weather anomalies (for up to 60 days), suggesting that stratospheric variability might be used to extend weather prediction beyond current time scales. In this paper, attention is drawn away from the stratosphere to demonstrate that the originating point of ESEs is located in the troposphere. First, it is shown that anomalously strong eddy heat fluxes at 100 $\mathrm{hPa}$ nearly always precede weak vortex events, and conversely, anomalously weak eddy heat fluxes precede strong vortex events, consistent with wave-mean flow interaction theory. This finding clarifies the dynamical nature of ESEs and suggests that a major source of stratospheric variability (and thus predictability) is located in the troposphere below and not in the stratosphere itself. Second, it is shown that the daily time series of eddy heat flux found at $100 \mathrm{hPa}$ and integrated over the prior 40 days, exhibit a remarkably high anticorrelation $(-0.8)$ with the Arctic Oscillation (AO) index at $10 \mathrm{hPa}$. Following Baldwin and Dunkerton, it is then demonstrated that events with anomalously strong (weak) integrated eddy heat fluxes at $100 \mathrm{hPa}$ are followed by anomalously large (small) surface values of the AO index up to 60 days following each event. This suggests that the stratosphere is unlikely to be the dominant source of the anomalous surface weather regimes discussed in Thompson et al.
\end{abstract}

\section{Introduction}

It has recently been suggested that knowledge of the state of the stratosphere might be useful for understanding tropospheric climate and extending weather forecasting beyond the current limit of about a week. This suggestion has been motivated by the fact that anomalous values in the Arctic Oscillation (AO) index (Thompson and Wallace 1998) are found to appear in the stratosphere first, and subsequently propagate downward over periods of several weeks (Baldwin and Dunkerton 1999). Moreover, it has been shown that extreme stratospheric events (ESEs), defined as days when the $\mathrm{AO}$ index exceeds a given threshold either positively or negatively, are followed by anomalous weather regimes at the surface that persist for up to two months (Baldwin and Dunkerton 2001, hereafter BD2001; Thompson et al. 2002).

The fact that extreme AO values arise in the upper stratosphere first, with no preceding or simultaneous

Corresponding author address: Dr. L. M. Polvani, Columbia University, 500 W. 120th Street, Room 216, New York, NY 10027. E-mail:1mp@columbia.edu large values in the tropospheric AO, gives the (misleading) impression that the ESEs originate in the upper stratosphere. Similarly, the fact that extreme AO values descend from the upper stratosphere to the troposphere has generated a fair amount of interest in the possibility that the stratosphere might be "forcing" the troposphere and thus contributing to climate variability. This, however, runs counter to our most basic understanding of the dynamics of planetary-scale waves and their interaction with stratospheric polar vortices. The main goal of this paper is to clarify the dynamical nature of ESEs, and to demonstrate that they originate in troposphere.

Since ESEs are defined on the basis of AO values (at $10 \mathrm{hPa}$ ), and since the AO is a very good proxy for the strength of the polar night jet in the stratosphere, it should be clear that ESEs correspond to instances of either anomalously weak or strong stratospheric polar vortices (e.g., stratospheric sudden warmings or coolings). A number of previous case studies have linked such events to anomalous wave activity entering the stratosphere. In particular, case studies have documented that large upward wave activity [Eliassen-Palm (E-P) flux] precedes the breakdown of the vortex in individual sudden warming events (Baldwin et al. 1989; Kodera 
and Chiba 1995; Naujokat et al. 2002; Palmer 1981), and that periods with an anomalously strong vortex are associated with weak wave activity entering the stratosphere (Coy et al. 1997; Pawson and Naujokat 1999). Similarly, a clear relationship has been established on interannual time scales between the state of the stratosphere and the time-integrated wave activity upwelling from the troposphere: winters with strong upward wave activity are associated with warmer polar temperatures, more ozone, and a polar vortex that is weaker and breaks up earlier (Fusco and Salby 1999; Waugh et al. 1999; Newman et al. 2001; Hu and Tung 2002).

However, a systematic analysis of the entire data record has not been performed, and the statistical robustness of the link between upward-propagating wave activity near the tropopause and the occurrence of extreme AO values (ESEs) in the stratosphere has not been tested. In this paper, we examine on daily time scales the relationship between the state of the stratosphere and upward wave activity. We demonstrate that there is a statistically significant correlation between the AO index at $10 \mathrm{hPa}$ and the upward wave activity entering the stratosphere integrated over the preceding month and that individual ESEs are nearly always preceded by anomalous upward wave activity. From this we deduce that ESEs originate in the troposphere, as suggested by Christiansen (2001).

We also show that upward wave activity near the tropopause can be used to construct composites of ESEs whose time-height structure is very similar to the one presented in BD2001 and, more importantly, that anomalous surface weather regimes are found over the 60 days following large anomalies in upward wave activity near the tropopause. Again, this indicates that the origin of anomalous surface weather regimes is to be found in the troposphere and not the stratosphere.

\section{Results}

A key aspect of this study is that we consider the time-integrated wave activity entering the stratosphere, rather than daily values (with a time lag), which have been used in most of the case studies mentioned above. There is theoretical support for this. Newman et al. (2001) have shown that stratospheric polar temperatures (and, via geostrophic balance, stratospheric winds) on a given day are related not to the instantaneous upward wave activity, but to its weighted integral over several weeks prior to that day. We here use National Centers for Environmental Prediction-National Center for Atmospheric Research (NCEP-NCAR) reanalyses (Kalnay et al. 1996) from 1958 to 2001 to examine, on a daily basis, the relationship between ESEs and wave activity entering the stratosphere. We calculate the meridional eddy heat flux $\overline{v^{\prime} T^{\prime}}$ at a specified level (usually $100 \mathrm{hPa}$ ) averaged between $45^{\circ}$ and $75^{\circ} \mathrm{N}$, and again averaged over $N$ (usually 40) days prior to each day. We average the heat flux between $45^{\circ}$ and $75^{\circ} \mathrm{N}$ to be

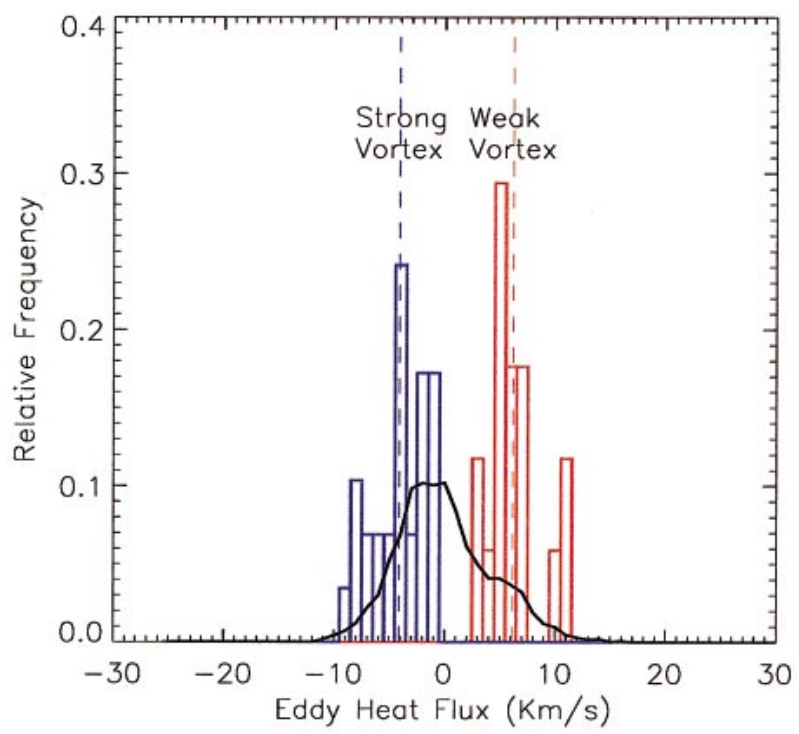

FIG. 1. Probability distribution functions for the 40-day averaged heat flux anomaly at $100 \mathrm{hPa}$ for all winter days (black curve), and the 18 weak vortex (red) and 30 strong vortex (blue) events, as defined in BD2001.

consistent with several previous studies that have considered this same quantity when examining interannual variations in stratospheric wave forcing (e.g., Waugh et al. 1999; Newman et al. 2001; Randel et al. 2002). Similar results are obtained if the vertical component of the E-P flux is used or if averaging occurs over a wider latitude range. To remove seasonal variations, the climatological mean for each day is removed (thus, all presented fluxes are anomalies). We refer to this quantity as the "averaged heat flux" and, unless otherwise stated, these fluxes are calculated at $100 \mathrm{hPa}$ and averaged over 40 days prior to a given day.

Considering the ESEs defined in BD2001, that is, those days in which the northern annular mode (NAM) index at $10 \mathrm{hPa}$ exceeds a positive or negative threshold, we ask the simple question: What is the upward wave activity flux preceding each event? The answer is shown in Fig. 1. For the 18 weak vortex events defined in BD2001, the probability distribution function (PDF) of averaged heat fluxes at $100 \mathrm{hPa}$ is shown by the red bars; the corresponding distribution for the 30 strong events is shown by the blue bars. Notice how well separated the PDFs of the weak and strong events are, and how they fall on either side of the black curve (all winter days).

From Fig. 1 it is clear that weak vortex events are preceded by anomalously strong wave activity entering the stratosphere. This is not surprising and offers a potentially simple dynamical explanation of these events: in the 40 days prior to each weak vortex (low NAM) event, anomalously large wave activity has propagated up from the troposphere and, breaking, has deposited its westerly momentum in the stratosphere, thus weak- 

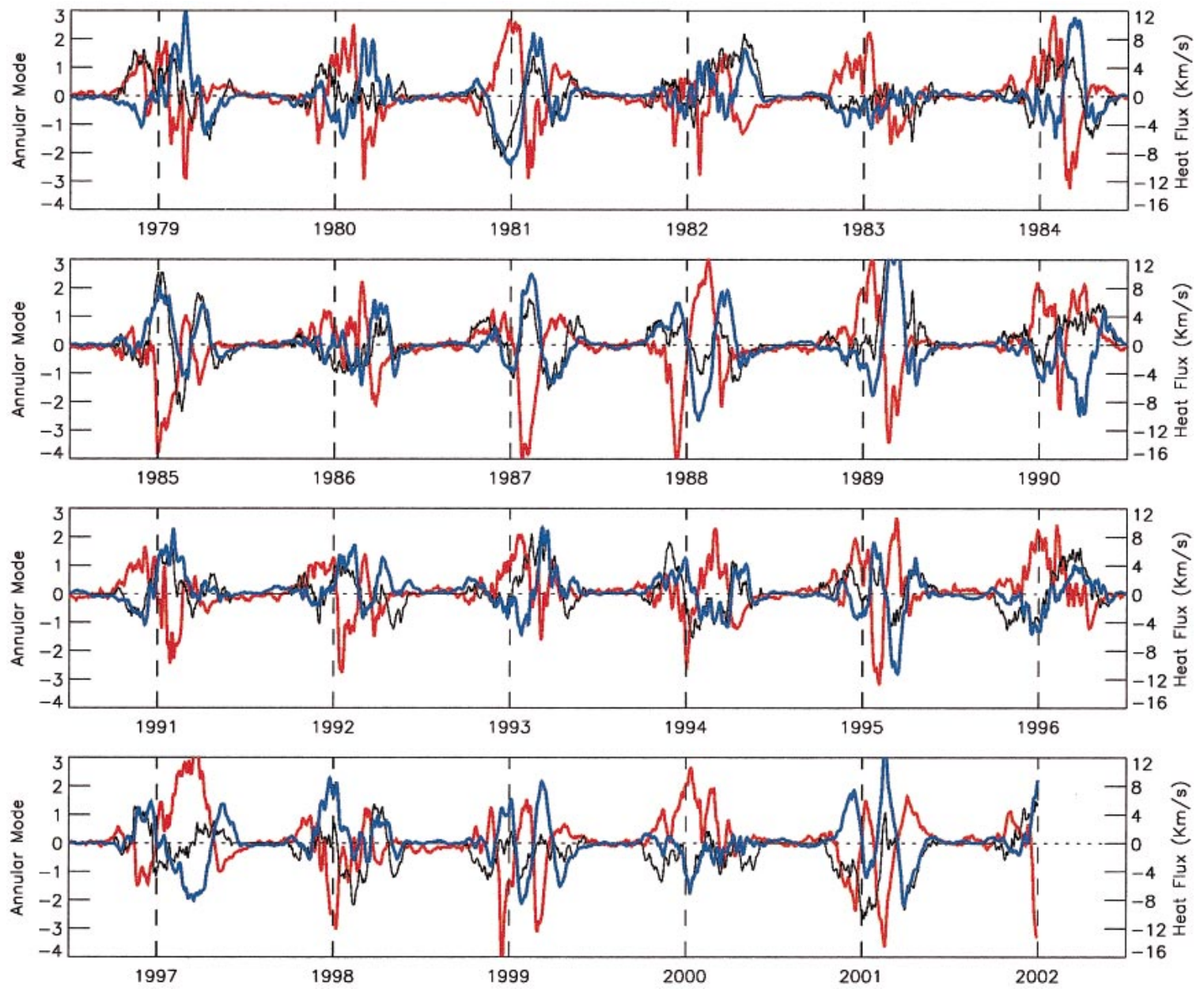

FIG. 2. Daily values of the NAM index at $10 \mathrm{hPa}$ (red) and 40-day averaged heat fluxes anomalies at 100 (blue) and $300 \mathrm{hPa}$ (black), for 1 Jul 1978-31 Dec 2002. Heat fluxes are averaged over 40 days up to the given day. The heat flux at $300 \mathrm{hPa}$ has been multiplied by a factor of 2. The dashed vertical lines mark 1 Jan of each year.

ening the polar vortex. Similarly, Fig. 1 shows that strong vortex (high NAM) events in the stratosphere are preceded by anomalously weak upward wave activity; again, this fact is in perfect consonance with our understanding of stratospheric dynamics.

It is interesting to note in Fig. 1 that the averaged heat flux preceeding ESEs (as defined in BD2001 on the basis of the NAM index at $10 \mathrm{hPa}$ ) is not symmetric. The histogram for the weak vortex (red) events is centered at roughly two standard deviations to the right of the PDF for all days, while the histogram from the strong vortex (blue) events is only about one standard deviation to the left. From the dynamical interpretation we have offered, one would not really expect symmetry. In some sense only the weak vortex ESEs are true "events," insofar as something has in fact happened (notably, a much larger than average upward wave propagation followed by wave breaking, typical of a sudden warming). On the other hand, when the vortex is anomalously strong, not much actually happens, that is, the wave activity entering the stratosphere is weak and thus fewer waves are propagating upwards, breaking and decelerating the vortex.

This dynamical interpretation offered on the basis of the PDFs alone is much strengthened by inspection of the actual time series. These are plotted in Fig. 2 for the last $24 \mathrm{yr}$ of the record. Notice the extremely high anticorrelation $(-0.8)$ between the NAM index at 10 $\mathrm{hPa}$ (red curve) and of the averaged heat flux at 100 $\mathrm{hPa}$ (blue curve). Such a high correlation lends strong support to our interpretation of upward wave activity fluxes as precursors to ESEs.

In Fig. 3, we explore the dependence of the correlation between the averaged heat flux at $100 \mathrm{hPa}$ and the NAM index at $10 \mathrm{hPa}$ on two parameters: the time interval over which the heat flux is averaged (the integration period) and the time lag between the end of the integration period and the time of the $10 \mathrm{hPa}$ NAM index. Figure 3 shows that the highest anticorrelations are found for integration periods of 20 days or more, 


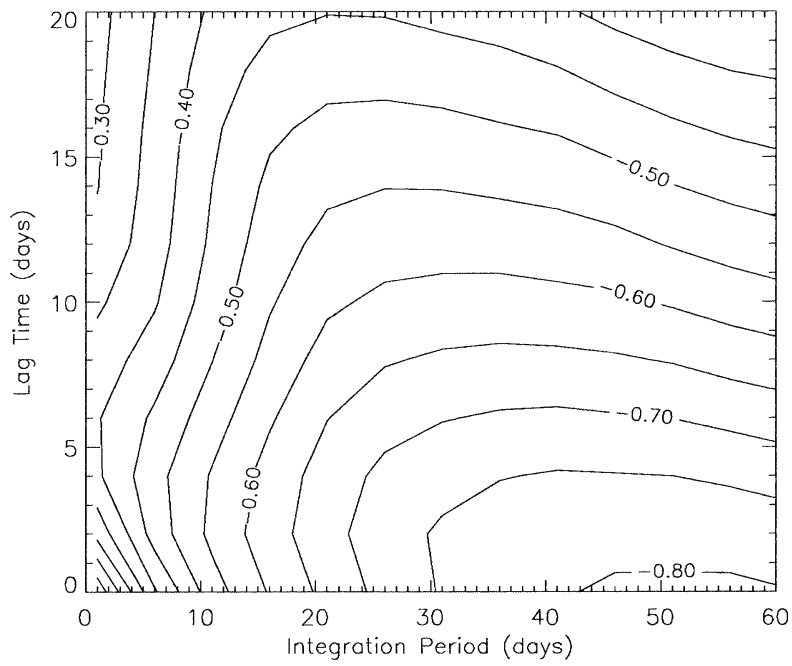

FIG. 3. The correlation coefficient between the NAM index at 10 $\mathrm{hPa}$ and the averaged heat flux at $100 \mathrm{hPa}$, as a function of the integration period for the heat flux and time lag between the end of that integration period and the time of the NAM index. The contour interval is 0.05. Data for all winters (Dec-Feb) from 1958 to 2001 are used to compute these correlations. with little sensitivity beyond that value. Note that high anticorrelations basically disappear for averaging periods over less than 10 days; this is why the anticorrelation using daily heat fluxes is much lower than that using heat fluxes averaged over 20 or more days [consistent with the Newman et al. (2001) theory]. This result holds even if a time lag is used between the daily heat fluxes at $100 \mathrm{hPa}$ and the NAM index at $10 \mathrm{hPa}$. The maximum correlation with the daily heat flux occurs for a time lag of 5 days, but this correlation is only around -0.4 .

In order to establish the tropospheric origin of ESEs, we have also explored how the correlation between the $10 \mathrm{hPa}$ NAM index and the upward heat flux depends on the level at which the heat flux is measured. The time series of averaged heat flux at $300 \mathrm{hPa}$ is actually shown in Fig. 2 (cf. the thin black line). There is, in general, good correspondence between anomalous events in the 100 and $300 \mathrm{hPa}$ heat flux time series, and the correlation of the two heat flux time series exceeds 0.55 [an even closer relationship holds between 100 and $200 \mathrm{hPa}$ (not shown) with correlation around 0.9]. These high correlations indicates that most of the variations in heat flux at $100 \mathrm{hPa}$ originate at $300 \mathrm{hPa}$ and lower, that is, the wave activity at $100 \mathrm{hPa}$ is of tropospheric origin.

The correlations between 200- or 300-hPa heat fluxes

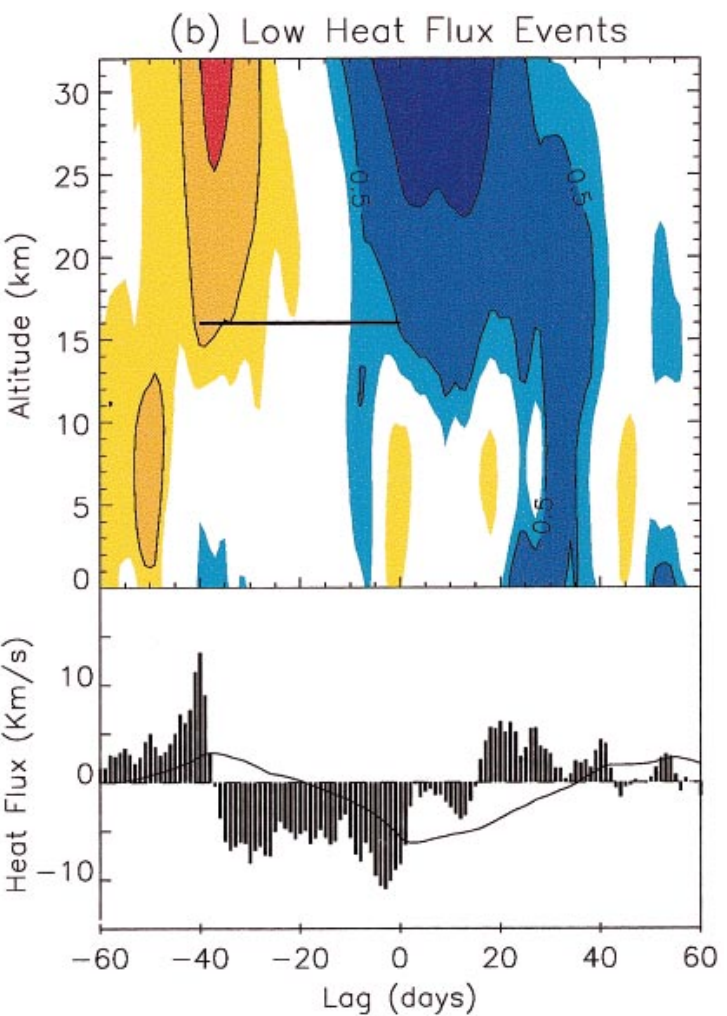

FIG. 4. (top) Composites of height-time development of the NAM index for (a) 25 high heat flux events and (b) 24 low heat flux events. The horizontal line marks the 40-day period over which the averaged heat flux is anomalous. Values greater than 0.25 and smaller than -0.25 are shaded, and values greater (smaller) than $0.5(-0.5)$ contoured, with a contour interval of 0.5 . (bottom) Temporal evolution of composite mean daily (bars) and 40-day averaged (curves) heat flux anomalies at $100 \mathrm{hPa}$. 


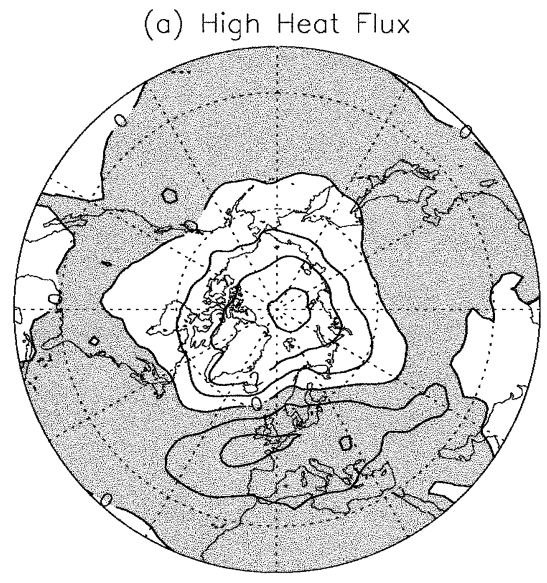

(b) Low Heat Flux



FIG. 5. Average sea level pressure anomalies for (a) 60 days following high heat flux events (1500 days) and (b) 60 days following low heat flux events (1440 days). The contour interval is $1 \mathrm{hPa}$, with negative values shaded.

with the NAM index at $10 \mathrm{hPa}$ show a similar dependence on averaging period and time lag as shown in Fig. 3, but with weaker correlations (the maximum anticorrelations at 200 and $300 \mathrm{hPa}$ are -0.55 and -0.3 , respectively). These reduced correlations suggest that although the wave activity propagating above $100 \mathrm{hPa}$ originates in the troposphere, the flow near the tropopause is important for determining how much wave activity makes it into the middle stratosphere. We are currently examining in more detail the propagation of wave activity within the 300- to $100-\mathrm{hPa}$ region.

Having demonstrated that ESEs are preceeded by anomalous upward wave flux from the troposphere, one might now ask whether events composited from thresholding the upward flux would look substantially different from those presented in BD2001. The composite NAM index of events selected by using a threshold of $\pm 5.5 \mathrm{~K} \mathrm{~m} \mathrm{~s}^{-1}$ on the 40 -day time-averaged heat flux at $100 \mathrm{hPa}$ are presented in the upper panels in Fig. 4. In Fig. 4's lower panels, we plot the composites of the daily values of upward wave activity at $100 \mathrm{hPa}$.

The upper panels in Fig. 4 are, qualitatively and quan-

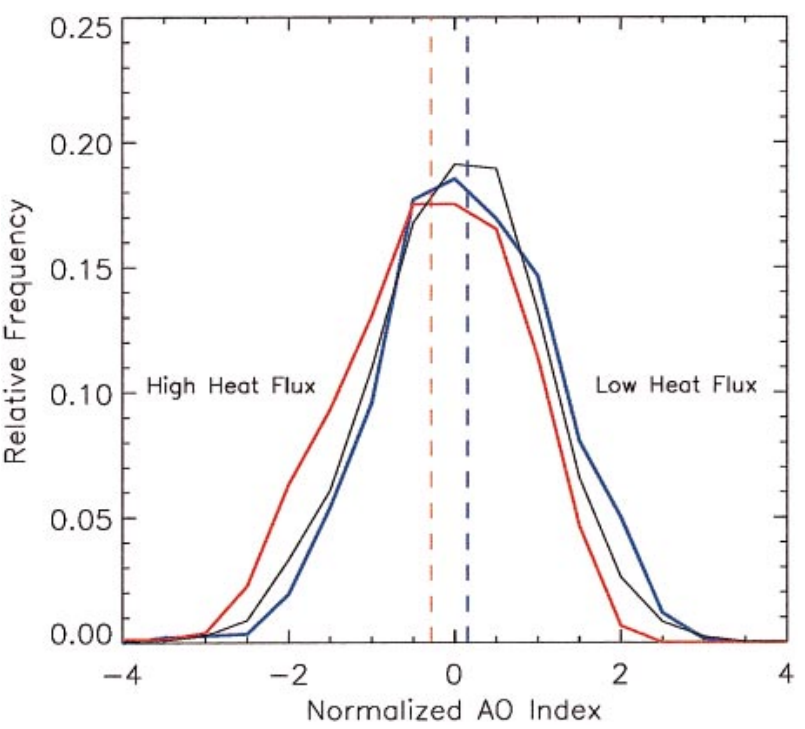

FIG. 6. Probability distribution functions for the normalized AO index at the surface during Dec-Apr (black curve), 60 days following high heat flux events (red), and 60 days following low heat flux events (blue).

titatively, very similar to those in Fig. 2 of BD2001. In particular, note how the NAM anomaly appears in the upper stratosphere first, and downward propagation of that anomaly follows. As the NAM index is very small at nearly all levels of the atmosphere during 10-20 days before the appearance of the NAM anomalies at $10 \mathrm{hPa}$, such plots, considered in isolation, might lead one to infer that a source of variability in the upper stratosphere is influencing the lower stratosphere and even the troposphere below. However, the fact that the upper-stratospheric NAM anomalies are preceded by anomalies in the upward wave activity, as the lower panels show, invalidates such a scenario.

An interesting feature of the lower panels in Fig. 4 is that the instantaneous heat fluxes change rapidly during the onset of ESEs, especially for weak vortex events where the flux decreases from very large values to very small values in a matter of days. The cause of this dramatic change (and whether it is due to conditions in the troposphere, stratosphere, or both) is unclear at present. However, this does show that care is required when choosing averaging periods to examine fluxes during ESEs.

Finally, BD2001 and Thompson et al. (2002) have shown that surface anomalies can be detected up to 60 days following ESEs. We will now show that, not surprisingly, surface anomalies of a nearly identical kind emerge when the upward wave activity at $100 \mathrm{hPa}$ is used to define anomalies.

We first plot, in Fig. 5, the mean sea level pressure anomalies averaged over the 60 days following (a) the 25 high heat flux events and (b) the 24 low heat flux events shown in the upper panels in Fig. 4. This figure is directly comparable to Fig. 3 of BD2001 and shows 
very similar features. We stress that no EOF was computed in producing this figure; we simply use surface pressure fields for 0-60 days following high and low heat fluxes at $100 \mathrm{hPa}$. Again, the inescapable conclusion to be drawn from this figure is that the stratosphere is not the ultimate location back to which surface weather regimes can be traced. The source of anomalous surface weather regimes is located in the troposphere itself.

Second, in Fig. 6, we show the PDF of surface AO for the climatology (black), as well as for the 2 months following the high (red) and low (blue) heat flux events, composited on the upward wave activity flux. There is a noticeable shift in the surface PDFs for 60 days after high or low heat flux events. Surface values of the AO index greater than 1.0 are nearly three times more likely following weak upward flux events than strong flux events, and similarly, values less than -1.0 are roughly three times more likely following strong rather than weak upward flux events. Figure 6 is very similar to Fig. 4a of BD2001, the key difference being that all the data needed to construct this figure are located at or below $100 \mathrm{hPa}$. In other words, anomalous upward wave activity near the tropopause manifests itself at the surface many weeks later.

\section{Discussion}

In summary, we have shown that anomalous low upward wave activity fluxes at $100 \mathrm{hPa}$ (and below) precede extreme stratospheric events and anomalous surface values of the AO up to 60 days later. Because the upward wave flux is associated with planetary-scale waves propagating from the troposphere to the stratosphere, our analysis clarifies the dynamical source of the extreme stratospheric events. In particular, it shows that the stratosphere is not the originating point of ESEs. More importantly, however, our analysis shows that anomalous surface weather regimes can be traced back not just to the upper stratosphere, as noted by Baldwin and Dunkerton (2001), but even further back in time to the troposphere itself. The key point that emerges from this study, therefore, is that the stratosphere is not the primary source of anomalous events. While the stratosphere surely plays a role in mediating and possibly modulating these events, their origin is to be found in the troposphere below.

What the precise role of the stratosphere is remains unclear at the moment. Is the stratosphere simply passively reacting to the changes in upward wave flux from the troposphere or does it play an active role in controlling the wave flux? In Fig. 1, one might argue that the anomalous events are just the tails of a Gaussian PDF. However there is some indication that the stratospheric state preceding the anomalous flux events is not random. Note in Fig. 4 how a strong polar vortex typically precedes the high upward flux event and vice versa. This suggests that while the source of the events may be located in the troposphere, the stratosphere could play some role in enhancing or suppressing the events.
A preliminary study with an idealized stratosphere-troposphere model, in which the tropospheric variability is suppressed by applying a strong relaxation there, shows that by modulating the upward wave flux near the tropopause, the stratosphere is able to internally generate alternating cycles of strong and weak polar winds (Scott and Polvani 2004), confirming earlier results with severely truncated models (Holton and Mass 1976; Yoden 1987; Scott and Haynes 2000). However, more work is needed to elucidate the precise roles of the troposphere and the stratosphere in determining the amount of upward wave activity entering the stratosphere, and thus the occurrence of anomalous surface weather regimes many weeks later.

Acknowledgments. The authors are grateful to Bill Randel, Paul Kushner, and Isaac Held for useful discussions and thank Mark Baldwin for the NAM indices, and Paul Newman and Eric Nash for access to the NCEP-NCAR reanalyses. This work is funded, in part, by grants from the National Science Foundation and the National Aeronautics and Space Administration.

\section{REFERENCES}

Baldwin, M. P., and T. J. Dunkerton, 1999: Propagation of the Arctic Oscillation from the stratosphere to the troposphere. J. Geophys. Res., 104, 30 937-30946.

—_ and —_, 2001: Stratospheric harbingers of anomalous weather regimes. Science, 294, 581-584.

—, X. Cheng, and T. Dunkerton, 1989: The stratospheric major warming of early December 1987. J. Atmos. Sci., 46, 2863-2884.

Christiansen, B., 2001: Downward propagation of zonal mean wind anomalies from the stratosphere to the troposphere: Model and reanalysis. J. Geophys. Res., 106, 27 307-27 322.

Coy, L., E. Nash, and P. Newman, 1997: Meteorology of the polar vortex: Spring 1997. Geophys. Res. Lett., 24, 2693-2696.

Fusco, A., and M. Salby, 1999: Interannual variations of total ozone and their relationship to variations of planetary wave activity. J. Climate, 12, 1619-1629.

Holton, J. R., and C. Mass, 1976: Stratospheric vacillation cycles. J. Atmos. Sci., 33, 2218-2225.

$\mathrm{Hu}, \mathrm{Y}$., and K. K. Tung, 2002: Interannual and decadal variations of planetary wave activity, stratospheric cooling, and the Northern Hemisphere annular mode. J. Climate, 15, 1659-1673.

Kalnay, E., and Coauthors, 1996: The NCEP/NCAR 40-Year Reanalysis Project. Bull. Amer. Meteor. Soc., 77, 437-471.

Kodera, K., and M. Chiba, 1995: Role of planetary waves in the stratosphere-troposphere coupled variability in the Northern Hemisphere winter. J. Geophys. Res., 100, $11055-11068$.

Naujokat, B., K. Krüger, K. Matthes, J. Hoffmann, M. Kunze, and K. Labitzke, 2002: The early major warming in December 2001-Exceptional? Geophys. Res. Lett., 29, 2023, doi:10.1029/ 2002 GL015316.

Newman, P. A., E. R. Nash, and J. Rosenfield, 2001: What controls the temperature of the Arctic stratosphere during the spring? $J$. Geophys. Res., 106, 19 999-20 010.

Palmer, T., 1981: Diagnostic study of a wavenumber-2 stratospheric sudden warming in a transformed Eulerian-mean formalism. $J$. Atmos. Sci., 38, 844-855.

Pawson, S., and B. Naujokat, 1999: The cold winters of the middle 1990 s in the northern lower stratosphere. J. Geophys. Res., 104, 14 209-14 222.

Randel, W. J., F. Wu, and R. Stolarski, 2002: Changes in column ozone correlated with the stratosphere EP flux. J. Meteor. Soc. Japan, 80, 849-862. 
Scott, R. K., and P. H. Haynes, 2000: Internal vacillations in stratosphere-only models. J. Atmos. Sci., 57, 2333-2350.

- , and L. M. Polvani, 2004: Stratospheric control of upward wave flux near the tropopause. Geophys. Res. Lett., 31, L02115, doi:10.1029/2003GL017965.

Thompson, D. W. J., and J. M. Wallace, 1998: The Arctic Oscillation signature in the wintertime geopotential height and temperature fields. Geophys. Res. Lett., 25, 1297-1300.
-, M. P. Baldwin, and J. M. Wallace, 2002: Stratospheric connection to Northern Hemisphere wintertime weather: Implications for predictions. J. Climate, 15, 1421-1428.

Waugh, D., W. Randel, S. Pawson, P. Newman, and E. Nash, 1999: Persistence of the lower stratospheric polar vortices. J. Geophys. Res., 104, 27 191-27 201.

Yoden, S., 1987: Dynamical aspects of stratospheric vacillations in a highly truncated model. J. Atmos. Sci., 44, 3683-3695. 